\title{
Variational Inequalities with Multivalued Lower Order Terms and Convex Functionals in Orlicz-Sobolev Spaces
}

\author{
Ge Dong ${ }^{1,2}$ and Xiaochun Fang ${ }^{1}$ \\ ${ }^{1}$ Department of Mathematics, Tongji University, Shanghai 200092, China \\ ${ }^{2}$ Department of Basic Teaching, Shanghai Jianqiao College, Shanghai 201319, China \\ Correspondence should be addressed to Xiaochun Fang; xfang@tongji.edu.cn
}

Received 12 January 2015; Accepted 27 February 2015

Academic Editor: Dumitru Motreanu

Copyright (c) 2015 G. Dong and X. Fang. This is an open access article distributed under the Creative Commons Attribution License, which permits unrestricted use, distribution, and reproduction in any medium, provided the original work is properly cited.

\begin{abstract}
We consider the existence of solutions of variational inequality form. Find $u \in D(J):\langle\mathscr{A}(u), v-u\rangle+\langle F(u), v-u\rangle+J(v)-J(u) \geq 0$, $\forall v \in W^{1} L_{M}(\Omega)$, whose principal part is having a growth not necessarily of polynomial type, where $\mathscr{A}$ is a second-order elliptic operator of Leray-Lions type, $F$ is a multivalued lower order term, and $J$ is a convex functional. We use subsupersolution methods to study the existence and enclosure of solutions in Orlicz-Sobolev spaces.
\end{abstract}

\section{Introduction}

Let $\Omega$ be a bounded domain in $\mathbb{R}^{N}(N \geq 1)$ with Lipschitz boundary, and let $\mathscr{A}(u)=-\operatorname{div} A(x, D u)$ be a Leray-Lions operator defined on $W^{1, p}(\Omega), p \in(1,+\infty)$.

Le [1] used subsupersolution methods to study the existence and enclosure of solutions of the variational inequality of the following form.

$\left(\mathrm{P}_{0}\right)$ Find $u \in D(J)$ such that

$$
\begin{aligned}
& \langle\mathscr{A}(u), v-u\rangle+\langle F(u), v-u\rangle \\
& \quad+J(v)-J(u) \geq 0, \quad \forall v \in W^{1, p}(\Omega),
\end{aligned}
$$

where $F$ is a multivalued lower order term and $J$ is a convex functional. Accordingly the function $A$ is supposed to satisfy polynomial growth conditions with respect to $D u$, where $D u$ is the derivative of $u$.

When trying to weaken this restriction on $A$, one is led to replace $W^{1, p}(\Omega)$ by a Sobolev space $W^{1} L_{M}(\Omega)$ built from an Orlicz space $L_{M}(\Omega)$ instead of $L^{p}(\Omega)$. Here the $N$-function $M$ which defines $L_{M}(\Omega)$ is related to the growth of the function A.
It is well known that, in the study of differential equations, different classes of differential equations correspond to different function space settings. The classical Sobolev space is a special case of Orlicz-Sobolev spaces.

In this paper, it is our purpose to study the existence and enclosure of solutions to the problem $\left(\mathrm{P}_{0}\right)$ in the setting of the Orlicz-Sobolev spaces.

The paper is organized as follows: Section 2 contains some preliminaries and some technical lemmas which will be needed in Section 3. In Section 3, we first establish some basic properties of the operator $\mathscr{A}$ in Orlicz-Sobolev spaces; next, following Le [1] in which a subsupersolution method for variational inequality of the form $\left(\mathrm{P}_{0}\right)$ in Sobolev spaces was established, we prove the existence and enclosure of solutions of the problem $\left(\mathrm{P}_{0}\right)$ in Orlicz-Sobolev spaces.

We refer to some results of a subsupersolution method for variational inequalities studied in variable exponent Sobolev spaces (cf., e.g., [2-4]) and partial differential equations in Musielak-Orlicz-Sobolev spaces (cf., e.g., [5]). For some classical results we also refer to [6-12].

\section{Preliminaries}

2.1. N-Function. Let $M: \mathbb{R}^{+} \rightarrow \mathbb{R}^{+}$be an $N$-function; that is, $M$ is continuous and convex, with $M(u)>0$ for $u>0$, 
$M(u) / u \rightarrow 0$ as $u \rightarrow 0$, and $M(u) / u \rightarrow+\infty$ as $u \rightarrow+\infty$. Equivalently, $M$ admits the representation $M(u)=\int_{0}^{u} p(t) d t$, where $p: \mathbb{R}^{+} \rightarrow \mathbb{R}^{+}$is a nondecreasing, right continuous function, with $p(0)=0, p(t)>0$ for $t>0$, and $p(t) \rightarrow+\infty$ as $t \rightarrow+\infty$.

The $N$-function $\bar{M}$ conjugated to $M$ is defined by $\bar{M}(v)=$ $\int_{0}^{v} q(s) d s$, where $q: \mathbb{R}^{+} \rightarrow \mathbb{R}^{+}$is given by $q(s)=\sup \{t:$ $p(t) \leq s\}$. tively.

$p, q$ are called the right-hand derivatives of $M, \bar{M}$, respec-

The $N$-function $M$ is said to satisfy the $\Delta_{2}$ condition near infinity ( $M \in \Delta_{2}$, for short), if for some $k>1$ and $\bar{u}>0$, $M(2 u) \leq k M(u), \forall u \geq \bar{u}$. It is readily seen that this will be the case if and only if for every $l>1$ there exists a positive constant $k=k(l)$ and $\tilde{u}>0$, such that $M(l u) \leq k M(u)$, $\forall u \geq \tilde{u}$, and equivalently, there exists $k_{0}>0$ and $u_{0}>0$, such that

$$
p(2 u) \leq k_{0} p(u), \quad \forall u \geq u_{0}
$$

(cf., e.g., [13]).

Moreover, one has the following Young inequality: $u v \leq$ $M(u)+\bar{M}(v), \forall u, v \geq 0$.

We will extend these $N$-functions into even functions on all $\mathbb{R}$.

Let $P, Q$ be two $N$-functions; we say that $P$ grows essentially less rapidly than $Q$ near infinity, denoted as $P \ll$ $Q$, if for every $\varepsilon>0, P(t) / Q(\varepsilon t) \rightarrow 0$ as $t \rightarrow+\infty$. This is the case if and only if $\lim _{t \rightarrow+\infty} Q^{-1}(t) / P^{-1}(t)=0$ (cf., e.g., $[14,15])$.

For a measurable function $u$ on $\Omega$, its modular is defined by $\rho_{M}(u)=\int_{\Omega} M(|u(x)|) d x$ (cf., e.g., [16]).

2.2. Orlicz Spaces. Let $\Omega$ be an open and bounded subset of $\mathbb{R}^{N}$ and $M$ an $N$-function. The Orlicz class $\mathscr{K}_{M}(\Omega)$ (resp., the Orlicz space $L_{M}(\Omega)$ ) is defined as the set of (equivalence classes of) real valued measurable functions $u$ on $\Omega$ such that

$$
\rho_{M}(u)<+\infty\left(\text { resp. } \rho_{M}\left(\frac{u}{\lambda}\right)<+\infty \text { for some } \lambda>0\right) .
$$

$L_{M}(\Omega)$ is a Banach space under the (Luxemburg) norm

$$
\|u\|_{(M)}=\inf \left\{\lambda>0: \rho_{M}\left(\frac{u}{\lambda}\right) \leq 1\right\}
$$

and $\mathscr{K}_{M}(\Omega)$ is a convex subset of $L_{M}(\Omega)$ but not necessarily a linear space.

The closure in $L_{M}(\Omega)$ of the set of bounded measurable functions with compact support in $\bar{\Omega}$ is denoted by $E_{M}(\Omega)$.

The equality $E_{M}(\Omega)=L_{M}(\Omega)$ holds if and only if $M \in$ $\Delta_{2}$; moreover, $L_{M}(\Omega)$ is separable.

$L_{M}(\Omega)$ is reflexive if and only if $M \in \Delta_{2}$ and $\bar{M} \in \Delta_{2}$ (cf., e.g., $[16])$.

Convergences in norm and in modular are equivalent if and only if $M \in \Delta_{2}$ (cf., e.g., [16]).

The dual space of $E_{M}(\Omega)$ can be identified with $L_{\bar{M}}(\Omega)$ by means of the pairing $\int_{\Omega} u(x) v(x) d x$, and the dual norm of $L_{\bar{M}}(\Omega)$ is equivalent to $\|\cdot\|_{(\bar{M})}($ cf., e.g., $[14,16])$.
2.3. Orlicz-Sobolev Spaces. We now turn to the OrliczSobolev space: $W^{1} L_{M}(\Omega)$ (resp., $W^{1} E_{M}(\Omega)$ ) is the space of all functions $u$ such that $u$ and its distributional partial derivatives lie in $L_{M}(\Omega)$ (resp., $E_{M}(\Omega)$ ). It is a Banach space under the norm

$$
\|u\|_{\Omega, M}=\sum_{|\alpha| \leq 1}\left\|D^{\alpha} u\right\|_{(M)}
$$

Denote $\|D u\|_{(M)}=\||D u|\|_{(M)}$ and $\|u\|_{1, M}=\|u\|_{(M)}+\|D u\|_{(M)}$. Clearly, $\|u\|_{1, M}$ is equivalent to $\|u\|_{\Omega, M}$.

Thus $W^{1} L_{M}(\Omega)$ and $W^{1} E_{M}(\Omega)$ can be identified with subspaces of the product of $N+1$ copies of $L_{M}(\Omega)$. Denoting this product by $\Pi L_{M}$, we will use the weak topologies $\sigma\left(\Pi L_{M}, \Pi E_{\bar{M}}\right)$ and $\sigma\left(\Pi L_{M}, \Pi L_{\bar{M}}\right)$ (cf. [15]).

If $M \in \Delta_{2}$, then $W^{1} L_{M}(\Omega)=W^{1} E_{M}(\Omega)$. If $M \in \Delta_{2}$ and $\bar{M} \in \Delta_{2}$, then $W^{1} L_{M}(\Omega)=W^{1} E_{M}(\Omega)$ are reflexive (cf., e.g., [14]); thus the weak topologies $\sigma\left(\Pi L_{M}, \Pi E_{\bar{M}}\right)$ and $\sigma\left(\Pi L_{M}, \Pi L_{\bar{M}}\right)$ are equivalent.

We recall the following notations and lemmas which will be used later.

Definition 1 (cf., e.g., [17, Definition 32.1]). Let $X, Y$ be nonempty sets, and $T: X \rightarrow 2^{Y}$ a multivalued mapping; that is, $T$ assigns to each point $u \in X$ a subset $T(u)$ of $Y$.

(i) The set $D(T)=\{u \in X: T(u) \neq \emptyset\}$ is called the effective domain of $T$.

(ii) The set $R(T)=\bigcup_{u \in X} T(u)$ is called the range of $T$.

(iii) The set $G(T)=\{(u, v) \in X \times Y: u \in D(T), v \in T(u)\}$ is called the graph of $T$.

Definition 2 (cf., e.g., [18, Definition 2.112]). Let $X, Y$ be Banach spaces. The multivalued operator $T: X \rightarrow 2^{Y}$ is called upper semicontinuous at $u_{0} \in X$, if for every open subset $V \subset Y$ with $T\left(u_{0}\right) \subset V$, a neighborhood $U\left(u_{0}\right)$ exists such that $T\left(U\left(u_{0}\right)\right) \subset V . T$ is called upper semicontinuous in $X$, if $T$ is upper semicontinuous at every $u_{0} \in X$.

The following definition can be referred to [19, Page 41], [18, Definition 2.120], [20, Definition 1], or [3, Definition 2.1(b)].

Definition 3. Let $X$ be a real reflexive Banach space. The operator $T: X \rightarrow 2^{X^{*}}$ is called pseudomonotone if the following conditions hold.

(i) The set $T(u)$ is nonempty, bounded, closed, and convex for all $u \in X$.

(ii) $T$ is upper semicontinuous from each finitedimensional subspace $V$ of $X$ to the weak topology on $X^{*}$.

(iii) If $\left\{u_{n}\right\} \subset X$ with $u_{n} \rightarrow u$ and if $u_{n}^{*} \in T\left(u_{n}\right)$ is such that $\lim \sup _{n \rightarrow \infty}\left\langle u_{n}^{*}, u_{n}-u\right\rangle \leq 0$, then, to each element $v \in X, u^{*}(v) \in T(u)$ exists with

$$
\liminf _{n \rightarrow \infty}\left\langle u_{n}^{*}, u_{n}-v\right\rangle \geq\left\langle u^{*}(v), u-v\right\rangle .
$$


Lemma 4 (see [21, Lemma 2.1]). If $u \in W^{1} L_{M}(\Omega)$, then $u^{+}, u^{-} \in W^{1} L_{M}(\Omega)$ and

$$
D u^{+}=\left\{\begin{array}{ll}
D u, & \text { if } u>0, \\
0, & \text { if } u \leq 0,
\end{array} \quad D u^{-}= \begin{cases}0, & \text { if } u \geq 0, \\
-D u, & \text { if } u<0 .\end{cases}\right.
$$

Here $u^{+}=\max \{u, 0\}, u^{-}=-\min \{u, 0\}$.

The following lemma can be referred to [18, Theorem 2.127] or [19, Theorem 2.11]. The proof of the lemma can be found in [19].

Lemma 5. Let $X$ be a real reflexive Banach space, $\Phi$ : $X \rightarrow 2^{X^{*}}$ a maximal monotone operator, and $u_{0} \in D(\Phi)$. Let $T: X \rightarrow 2^{X^{*}}$ be a pseudomonotone operator, and assume that either $T\left(u_{0}\right)$ is quasibounded or $\Phi\left(u_{0}\right)$ is strongly quasibounded. Assume further that $T: X \rightarrow 2^{X^{*}}$ is $u_{0^{-}}$ coercive; that is, a real-valued function $c: \mathbb{R}^{+} \rightarrow \mathbb{R}$ exists with $c(r) \rightarrow+\infty$ as $r \rightarrow+\infty$ such that, for all $\left(u, u^{*}\right) \in \operatorname{Gr}(T)$, we have $\left\langle u^{*}, u-u_{0}\right\rangle \geq c\left(\|u\|_{X}\right)\|u\|_{X}$. Then $T+\Phi$ is surjective; that is, $\operatorname{range}(T+\Phi)=X^{*}$.

\section{Main Results}

Let $\Omega$ be a bounded domain in $\mathbb{R}^{N}(N \geq 1)$ with Lipschitz boundary, $M$ an $N$-function, and $\bar{M}$ the complementary function of $M$. We say that $M$ satisfies $\left(M_{0}\right)$, if both $M$ and $\bar{M}$ satisfy the $\Delta_{2}$ condition near infinity, and $M$ satisfies the following coerciveness condition.

There exists a function $g:(0,+\infty) \rightarrow \mathbb{R}$ such that $g(s) \rightarrow+\infty$ as $s \rightarrow+\infty$ and

$$
M(s u) \geq s g(s) M(u), \quad \text { for } u \in \mathbb{R}, s>0 .
$$

Let $P$ be an $N$-function such that $P \ll M_{*}$, where $M_{*}$ is the Sobolev conjugate of $M$. Assume that $P$ and $\bar{P}$ satisfy the $\Delta_{2}$ condition near infinity.

In what follows we denote by $L^{0}(\Omega)$ the set of all (equivalence classes of) Lebesgue measurable functions from $\Omega$ to $\mathbb{R}$.

We consider the following variational inequality.

(P) Find $u \in D(J)$ such that

$$
\begin{aligned}
& \langle\mathscr{A}(u), v-u\rangle+\langle F(u), v-u\rangle \\
& \quad+J(v)-J(u) \geq 0, \quad \forall v \in W^{1} L_{M}(\Omega) .
\end{aligned}
$$

Detailed assumptions on $\mathscr{A}, F, J$ together with a precise formulation of this inequality are presented in the following subsection.

3.1. Assumptions and Problem Settings. Let $A: \Omega \times \mathbb{R}^{N} \rightarrow$ $\mathbb{R}^{N}$ be a Carathéodory function satisfying the following conditions:

(A1) For a.e. $x \in \Omega$ and all $\xi \in \mathbb{R}^{N}$

$$
\begin{gathered}
|A(x, \xi)| \leq b_{1} \bar{M}^{-1} M(|\xi|)+a_{1}(x), \\
A(x, \xi) \xi \geq b_{2} M(|\xi|)-a_{2}(x),
\end{gathered}
$$

where $b_{1}, b_{2}>0, a_{1} \in L_{\bar{M}}(\Omega)$, and $a_{2} \in L^{1}(\Omega)$.
(A2) $A$ is monotone in the following sense:

$$
\left[A\left(x, \xi_{1}\right)-A\left(x, \xi_{2}\right)\right]\left(\xi_{1}-\xi_{2}\right) \geq 0,
$$

for a.e. $x \in \Omega$ and all $\xi_{1}, \xi_{2} \in \mathbb{R}^{N}$.

Define $\mathscr{A}: W^{1} L_{M}(\Omega) \rightarrow\left[W^{1} L_{M}(\Omega)\right]^{*}$ by

$$
\begin{array}{r}
\langle\mathscr{A}(u), v\rangle=\int_{\Omega} A(x, D u(x)) \nabla v(x) d x, \\
u, v \in W^{1} L_{M}(\Omega) .
\end{array}
$$

Example 6. (1) Let $M(u)=|u|^{p} \log (1+|u|)$ for $u \in \mathbb{R}$, where $p>1$. Then it can be verified that $M$ and $\bar{M}$ are $N$-functions satisfying $\Delta_{2}$ condition near infinity and $M$ satisfies (8).

(2) Put $A(x, \xi)=\left(M(|\xi|) /|\xi|^{2}\right) \xi, \forall x \in \Omega, \xi \in \mathbb{R}^{N} \backslash\{0\}$. Then $A$ satisfies (A1)-(A2).

Remark 7. By (2), we have $p(u) \leq k_{0}(M(u) / u) \leq$ $k_{0} \bar{M}^{-1}(M(u)), \forall u \geq u_{0}$, for some $k_{0}>0$ and $u_{0}>0$.

Moreover, we have the following lemma.

Lemma 8. (1) Let $M \in \Delta_{2}$ and let A satisfy (A1)-(A2). Then the operator $A$ is well-defined, bounded, and monotone.

(2) Let $M \in \Delta_{2}$ and $\bar{M} \in \Delta_{2}$ and let $A$ satisfy (A1)(A2). Then the operator $\mathscr{A}$ is continuous. Moreover, $\mathscr{A}$ is pseudomonotone.

Proof. (1) For every $u \in W^{1} L_{M}(\Omega)$, by (10) and the convexity of $\bar{M}$, we have

$$
\begin{aligned}
\rho_{\bar{M}}\left(\frac{1}{\beta}|A(x, D u)|\right) \leq & \frac{1}{2} \int_{\Omega} \bar{M}\left(\bar{M}^{-1}(M(|D u|))\right) d x \\
& +\frac{1}{2} \int_{\Omega} \bar{M}\left(a_{1}(x)\right) d x \\
= & \frac{1}{2} \int_{\Omega} M(|D u|) d x \\
& +\frac{1}{2} \int_{\Omega} \bar{M}\left(a_{1}(x)\right) d x<\infty,
\end{aligned}
$$

for some $K>0$, where $\beta=\max \left\{2 b_{1}, 2\right\}$. Therefore, $A(x, D u) \in L_{\bar{M}}(\Omega)$. Then $\mathscr{A}$ is well-defined.

From (14), one has that $\left\{\|A(x, D u)\|_{(\bar{M})}: u \in E\right\}$ is bounded, for any bounded set $E \subset W^{1} L_{M}(\Omega)$.

The monotonicity of $\mathscr{A}$ follows from (12).

(2) To prove the continuity of $\mathscr{A}$, let

$$
u_{n} \longrightarrow u \text { in } W^{1} L_{M}(\Omega) \text {. }
$$

We will prove that $\mathscr{A}\left(u_{n}\right) \rightarrow \mathscr{A}(u)$ in $\left[W^{1} L_{M}(\Omega)\right]^{*}$, which is equivalent to that $\left\|A\left(x, D u_{n}\right)-A(x, D u)\right\|_{(\bar{M})} \rightarrow 0$, or

$$
\int_{\Omega} \bar{M}\left(\left|A\left(x, D u_{n}\right)-A(x, D u)\right|\right) d x \longrightarrow 0 .
$$


Assume that there exists $\varepsilon_{0}>0$ such that

$$
\int_{\Omega} \bar{M}\left(\left|A\left(x, D u_{n}\right)-A(x, D u)\right|\right) d x \geq \varepsilon_{0}, \quad \forall n .
$$

In view of (15) and by [22, Chapter IV, Section 3, Theorem 3] there exists a subsequence of $\left\{u_{n}\right\}$ still denoted by $\left\{u_{n}\right\}$ and $g \in L^{1}(\Omega)$ such that $D u_{n}(x) \rightarrow D u(x)$ and $M\left(\mid D u_{n}(x)-\right.$ $D u(x) \mid) \leq g(x)$ for a.e. $x \in \Omega$. Since $A$ is a Carathéodory function,

$\bar{M}\left(\left|A\left(x, D u_{n}(x)\right)-A(x, D u(x))\right|\right) \longrightarrow 0 \quad$ for a.e. $x \in \Omega$.

By (10) and the convexity and $\Delta_{2}$-property of $M$, we obtain that $M\left(\left|D u_{n}(x)\right|\right) \leq(K / 2) M\left(\left|D u_{n}(x)-D u(x)\right|\right)+$ $(K / 2) M(|D u(x)|) \leq(K / 2) g(x)+(K / 2) M(|D u(x)|)$, for some $K>0$. Therefore,

$$
\begin{aligned}
& \bar{M}\left(\left|A\left(x, D u_{n}(x)\right)-A(x, D u(x))\right|\right) \\
& \leq \frac{1}{2} \bar{M}\left(2\left|A\left(x, D u_{n}(x)\right)\right|\right) \\
&+\frac{1}{2} \bar{M}(2|A(x, D u(x))|) \\
& \leq C\left[M\left(\left|D u_{n}(x)\right|\right)+M(|D u(x)|)+\bar{M}\left(a_{1}(x)\right)\right] \\
& \leq C\left[g(x)+M(|D u(x)|)+\bar{M}\left(a_{1}(x)\right)\right],
\end{aligned}
$$

where $C>0$ is a constant. By Lebesgue's dominated convergence theorem, we get

$$
\int_{\Omega} \bar{M}\left(\left|A\left(x, D u_{n}\right)-A(x, D u)\right|\right) d x \longrightarrow 0 .
$$

This is a contradiction. Hence (16) holds. This shows that $\mathscr{A}$ is continuous.

Since $\mathscr{A}$ is monotone, hemicontinuous (in fact, continuous), and bounded on $W^{1} L_{M}(\Omega)$, it is pseudomonotone.

Let $J: W^{1} L_{M}(\Omega) \rightarrow \mathbb{R} \cup\{+\infty\}$ be a convex, proper (cf. [19]), lower semicontinuous function. The effective domain of $J$ is $D(J)=\left\{v \in W^{1} L_{M}(\Omega): J(v)<+\infty\right\}$, and let $\partial J$ be the subdifferential of $J$.

For any topological vector space $Z$, we use the notation $\mathscr{K}(Z)=\{E \subset Z: E \neq \emptyset, E$ is closed and convex $\}$. Let $f$ be a function from $\Omega \times \mathbb{R}$ to $\mathscr{K}(\mathbb{R})$ such that

(F1) $f$ is superpositionally measurable (cf. $[1,23]$ or [24]);

(F2) for a.e. $x \in \Omega$, the function $f(x, \cdot): \mathbb{R} \rightarrow \mathscr{K}(\mathbb{R})$ is upper semicontinuous.

The lower order term $F(u)$ is defined by the (multivalued) integral

$$
\langle F(u), v\rangle=\int_{\Omega} \tilde{f}(u) v d x, \quad \text { for } u, v \in W^{1} L_{M}(\Omega) .
$$

For a precise definition of $F$ and an interpretation of this integral, we need some further notations. For any $u \in L^{0}(\Omega)$, let $\widetilde{f}(u)$ be the set of all measurable selections of $f(\cdot, u(\cdot))$. We know that $\tilde{f}(u) \neq \emptyset$ since $f(\cdot, u(\cdot))$ is measurable.
3.2. A Subsupersolution Method. For $u, v \in L^{0}(\Omega)$, we use the standard notation $u \wedge v=\min \{u, v\}, u \vee v=\max \{u, v\}, u^{+}:=$ $u \vee 0, u^{-}:=-u \wedge 0$.

By Lemma $4, W^{1} L_{M}(\Omega)$ is closed under $\vee$ and $\wedge$. In fact, since $u \vee v=v+(u-v)^{+}$and $u \wedge v=v-(u-v)^{-}, u \vee v, u \wedge v \in$ $W^{1} L_{M}(\Omega)$, for any $u, v \in W^{1} L_{M}(\Omega)$.

Let $\Gamma=\Gamma\left(W^{1} L_{M}(\Omega)\right):=\left\{\phi: W^{1} L_{M}(\Omega) \rightarrow \mathbb{R} \cup\{\infty\}:\right.$ $\left.D(\phi)=\left\{v \in W^{1} L_{M}(\Omega): \phi(v) \neq \infty\right\} \neq \emptyset\right\}$ be the set of all proper (not necessarily convex) functionals from $W^{1} L_{M}(\Omega)$ to $\mathbb{R} \cup\{\infty\}$.

We introduce the following definitions as in [1].

Definition 9. Let $\phi, \psi \in \Gamma$. We say that $\phi \preceq \psi$ (or equivalently $\psi \geq \phi)$ if and only if $\phi(u \wedge v)+\psi(u \vee v) \leq \phi(u)+\psi(v)$, $\forall u, v \in W^{1} L_{M}(\Omega)$. $[1])$.

From Definition 9, if $\phi \preceq \psi$, then $D(\phi) \preceq D(\psi)$ (cf. e.g.

Definition 10. (1) $u \in W^{1} L_{M}(\Omega)$ is called a solution of (9) if $u \in D(J)$ and there exists $P \ll M_{*}$ and $\eta \in L_{\bar{P}}(\Omega)$ such that $\eta(x) \in f(x, u(x))$, for a.e. $x \in \Omega$, and

$$
\begin{aligned}
\int_{\Omega} A(x, D u)(\nabla v-D u) d x \\
\quad+\int_{\Omega} \eta(v-u) d x+J(v)-J(u) \geq 0
\end{aligned}
$$

$$
\forall v \in W^{1} L_{M}(\Omega)
$$

(2) $\underline{u} \in W^{1} L_{M}(\Omega)$ is called a subsolution of (9) if there exist $P \ll M_{*}, \eta \in L_{\bar{P}}(\Omega)$, and $\underline{J} \in \Gamma$ such that $\underline{J} \preceq J, \underline{u} \in D(\underline{J})$, $\underline{\eta}(x) \in f(x, \underline{u}(\bar{x}))$, for a.e. $x \in \bar{\Omega}$, and

$$
\begin{aligned}
\int_{\Omega} A(x, \nabla \underline{u})(\nabla v-\nabla \underline{u}) d x \\
\quad+\int_{\Omega} \underline{\eta}(v-\underline{u}) d x+\underline{J}(v)-\underline{J}(\underline{u}) \geq 0,
\end{aligned}
$$

for all $v \in \underline{u} \wedge D(J)$.

(3) $\bar{u} \in W^{1} L_{M}(\Omega)$ is called a supersolution of (9) if there exists $P \ll M_{*}, \bar{\eta} \in L_{\bar{P}}(\Omega)$, and $\bar{J} \in \Gamma$ such that $\bar{J} \geq J$, $\bar{u} \in D(\bar{J}), \bar{\eta}(x) \in f(x, \bar{u}(x))$, for a.e. $x \in \Omega$, and

$$
\begin{aligned}
\int_{\Omega} A(x, \nabla \bar{u})(\nabla v-\nabla \bar{u}) d x & \\
& +\int_{\Omega} \bar{\eta}(v-\bar{u}) d x+\bar{J}(v)-\bar{J}(\bar{u}) \geq 0
\end{aligned}
$$

for all $v \in \bar{u} \vee D(J)$.

From Definition 10, we see that $u$ is a solution of (9) if and only if $u$ is a solution of the following inclusion: find $u \in D(J)$ such that

$$
\mathscr{A}(u)+F(u)+\partial J(u) \ni 0 .
$$

We will study the existence and some properties of solutions of (9). The proof of the following theorem is based 
on the ideas and arguments in the proof of Proposition 2.2 in [4], Theorem 2.11 in [1], and Theorem 4.2 in [2] and is divided into several steps. Le [1] points that if $f$ is graph measurable then $f$ is superpositionally measurable. It is only needed in our proof $f$ is superpositionally measurable as [1]. However, we do not need the condition (F3) in [1].

Theorem 11. Let $M, A$, and $f$ satisfy $\left(M_{0}\right),(A 1)-(A 2)$, and (F1)-(F2), respectively. Assume that there are subsolutions $\underline{u}_{1}, \underline{u}_{2}, \ldots, \underline{u}_{k}$ and supersolutions $\bar{u}_{1}, \bar{u}_{2}, \ldots, \bar{u}_{m}$ of (9) such that

$$
\underline{u}:=\underline{u}_{1} \vee \underline{u}_{2} \vee \cdots \vee \underline{u}_{k} \leq \bar{u}:=\bar{u}_{1} \wedge \bar{u}_{2} \wedge \cdots \wedge \bar{u}_{m}
$$

and there exists $h \in L_{\bar{P}}(\Omega)$ such that

$$
\sup \{|\xi|: \xi \in f(x, u)\} \leq h(x) \text {, }
$$

for a.e. $x \in \Omega$, all $u \in[\underline{u}(x), \bar{u}(x)]$.

Then, there exists a solution of (9) such that

$$
\underline{u} \leq u \leq \bar{u} \text { a.e. in } \Omega \text {. }
$$

Proof. Denote $X=W^{1} L_{M}(\Omega)$. Since $P \ll M_{*}, X \hookrightarrow L_{P}(\Omega)$ is compact ([25, Theorem 2.2]). By Proposition 2.1 in [25], $M \ll M_{*}$. Hence, $X \hookrightarrow L_{M}(\Omega)$ is compact. We will use $i_{P}$ and $i_{M}$ for the embeddings from $X$ into $L_{P}(\Omega)$ and $L_{M}(\Omega)$, respectively. Let $i_{P}^{*}: L_{\bar{P}}(\Omega)=\left(L_{P}(\Omega)\right)^{*} \rightarrow X^{*}$ and $i_{M}^{*}$ : $L_{\bar{M}}(\Omega)=\left(L_{M}(\Omega)\right)^{*} \rightarrow X^{*}$ be the adjoints of $i_{P}$ and $i_{M}$, respectively. Thus $i_{P}, i_{M}, i_{P}^{*}$, and $i_{M}^{*}$ are compact. Note that $i_{P}$ is the usual identity embedding, $i_{P}(u)=u$ for $u \in X$; that is, $i_{P}(u)(x)=u(x)$ for a.e. $x \in \Omega$. Similarly, for $\eta \in L_{\bar{P}}(\Omega)$, $i_{P}^{*}(\eta)=\left.\eta\right|_{X^{*}}$.

Step 1 . Let $\underline{u}_{i}, \underline{\eta}_{i}(1 \leq i \leq k)$ and $\bar{u}_{j}, \bar{\eta}_{j}(1 \leq j \leq m)$ satisfy the conditions as in Definition 10 of sub- and supersolutions. Let $\Omega_{1}=\left\{x \in \Omega: \underline{u}(x)=\underline{u}_{1}(x)\right\}$, and

$$
\Omega_{i}=\left\{x \in \Omega \backslash \bigcup_{l=1}^{i-1} \Omega_{l}: \underline{u}(x)=\underline{u}_{i}(x)\right\},
$$

for $i=2, \ldots, k$. Similarly, let $\Omega^{1}=\left\{x \in \Omega: \bar{u}(x)=\bar{u}_{1}(x)\right\}$, and

$$
\Omega^{j}=\left\{x \in \Omega \backslash \bigcup_{l=1}^{j-1} \Omega^{l}: \bar{u}(x)=\bar{u}_{j}(x)\right\},
$$

for $j=2, \ldots, m$. Then $\Omega=\bigcup_{i=1}^{k} \Omega_{i}=\bigcup_{j=1}^{m} \Omega^{j}$. Define $\underline{\eta}=\sum_{i=1}^{k} \underline{\eta}_{i} \chi_{\Omega_{i}}$ and $\bar{\eta}=\sum_{j=1}^{m} \bar{\eta}_{j} \chi_{\Omega^{j}}$, where $\chi_{E}(E \subset \Omega)$ is the characteristic function of $E$. It is clear that $\eta, \bar{\eta} \in L_{\bar{p}}(\Omega)$ and $\underline{\eta}(x) \in f(x, \underline{u}(x)), \bar{\eta}(x) \in f(x, \bar{u}(x))$ for a.e. $\bar{x} \in \Omega$.

For $x \in \Omega, u \in \mathbb{R}$, put

$$
f_{0}(x, u)= \begin{cases}\{\underline{\eta}(x)\}, & \text { if } u<\underline{u}(x) \\ f(x, u), & \text { if } \underline{u}(x) \leq u \leq \bar{u}(x) \\ \{\bar{\eta}(x)\}, & \text { if } u>\bar{u}(x)\end{cases}
$$

Then, as in $[1,26]$, we can check that $f_{0}$ satisfies (F1) and (F2). From (31) and (27), we see that

$$
\sup \left\{|\xi|: \xi \in f_{0}(x, u)\right\} \leq h(x), \quad \text { a.e. } x \in \Omega, \forall u \in \mathbb{R} \text {. }
$$

We define $b: \Omega \times \mathbb{R} \rightarrow \mathbb{R}$ given by

$$
b(x, u)= \begin{cases}\bar{M}^{-1} M(u-\bar{u}(x)), & \text { if } u>\bar{u}(x), \\ 0, & \text { if } \underline{u}(x) \leq u \leq \bar{u}(x), \\ -\bar{M}^{-1} M(\underline{u}(x)-u), & \text { if } u<\underline{u}(x),\end{cases}
$$

for $x \in \Omega, u \in \mathbb{R}$. Then $b$ is a Carathéodory function and

$$
|b(x, u)| \leq a_{3}(x)+\bar{M}^{-1} M(2|u|)
$$

with $a_{3}(x)=\bar{M}^{-1} M(2|\underline{u}(x)|)+\bar{M}^{-1} M(2|\bar{u}(x)|) \in L_{\bar{M}}(\Omega)$. By Young inequality and the convexity of $M$, the operator $\mathscr{B}$ : $L_{M}(\Omega) \rightarrow L_{\bar{M}}(\Omega)$ defined by

$$
\begin{array}{r}
\langle\mathscr{B}(u), v\rangle_{L_{\bar{M}}(\Omega), L_{M}(\Omega)}=\int_{\Omega} b(x, u) v d x, \\
\forall u, v \in L_{M}(\Omega),
\end{array}
$$

is well defined. From (34), we see that $\mathscr{B}$ is a bounded operator. Moreover, the mapping $u \mapsto b(\cdot, u)$ is continuous from $L_{M}(\Omega)$ to $L_{\bar{M}}(\Omega)$. In fact, let $u_{n} \rightarrow u$ in $L_{M}(\Omega)$, as $n \rightarrow \infty$. If $u \mapsto b(\cdot, u)$ is not continuous, then there exists $\varepsilon_{0}>0$ such that $\int_{\Omega} \bar{M}\left(\left|b\left(x, u_{n}(x)\right)-b(x, u(x))\right|\right) d x \geq$ $\varepsilon_{0}, \forall n \in \mathbb{N}$. By passing to a subsequence, if necessary, we have $u_{n}(x) \rightarrow u(x)$ a.e. $x \in \Omega$ and there is $g_{1} \in L^{1}(\Omega)$ such that $M\left(\left|u_{n}(x)-u(x)\right|\right) \leq g_{1}(x)$ for a.e. $x \in \Omega$. Then $\bar{M}\left(\left|b\left(x, u_{n}(x)\right)-b(x, u(x))\right|\right) \rightarrow 0$ a.e. $x \in \Omega$, as $n \rightarrow \infty$. Since $\bar{M}$ satisfies $\Delta_{2}$ condition and is convex,

$$
\begin{aligned}
\bar{M}\left(\left|b\left(x, u_{n}(x)\right)-b(x, u(x))\right|\right) \\
\leq \frac{K}{2} \bar{M}\left(\left|b\left(x, u_{n}(x)\right)\right|\right) \\
\quad+\frac{K}{2} \bar{M}(|b(x, u(x))|) \\
\leq C\left[M(|u(x)|)+M\left(\left|u_{n}(x)\right|\right)+\bar{M}\left(a_{3}(x)\right)\right] \\
\leq C\left[M(|u(x)|)+g_{1}(x)+\bar{M}\left(a_{3}(x)\right)\right],
\end{aligned}
$$

where $C>0$ and the function in the right-hand side belongs to $L^{1}(\Omega)$. Using Lebesgue's dominated convergence theorem, $\int_{\Omega} \bar{M}\left(\left|b\left(x, u_{n}(x)\right)-b(x, u(x))\right|\right) d x \rightarrow 0$, as $n \rightarrow \infty$. This is a contradiction. Due to the compact embedding $X \hookrightarrow$ $L_{M}(\Omega), i_{M}^{*} \mathscr{B} i_{M}$ is weakly-strongly continuous from $X$ into its dual space $X^{*}$. It follows that $i_{M}^{*} \mathscr{B} i_{M}$ is a (single-valued) pseudomonotone operator from $X$ into $X^{*}$. 
For $x \in \Omega$ and $u \in \mathbb{R}$, define $T_{i}(x, u)=\left|\underline{\eta}_{i}(x)-\underline{\eta}(x)\right| \sigma((u-$ $\left.\left.\underline{u}_{i}(x)\right) /\left(\underline{u}(x)-\underline{u}_{i}(x)\right)\right)(1 \leq i \leq k)$ and $T^{\bar{j}}(x, u)=\mid \bar{\eta}_{j}(x)-$ $\bar{\eta}(x) \mid\left[1-\sigma\left((u-\bar{u}(x)) /\left(\bar{u}_{j}(x)-\bar{u}(x)\right)\right)\right](1 \leq j \leq m)$, where

$$
\sigma(s)= \begin{cases}1, & s<0, \\ 1-s, & 0 \leq s \leq 1, \\ 0, & s>1 .\end{cases}
$$

Since $X \hookrightarrow L_{P}(\Omega)$ is compact, the mapping $i_{P}^{*} \mathscr{T}_{i} i_{P}$ and mapping $i_{P}^{*} \mathscr{T}^{j} i_{P}$ are weakly-strongly continuous and bounded. Consequently, $i_{P}^{*} \mathscr{T}_{i} i_{P}$ and $i_{P}^{*} \mathscr{T}^{j} i_{P}$ are (singlevalued) pseudomonotone operators from $X$ into $X^{*}$.

Next, we will find $u \in D(J)$ and $\widetilde{\eta} \in\left(i_{P}^{*} \tilde{f}_{0} i_{P}\right)(u)$ such that

$$
\begin{aligned}
& \left\langle\mathscr{A}(u)+\tilde{\eta}+\left(i_{M}^{*} \mathscr{B} i_{M}\right)(u)-\sum_{i=1}^{k}\left(i_{P}^{*} \mathscr{T}_{i} i_{P}\right)(u)\right. \\
& \left.\quad+\sum_{j=1}^{m}\left(i_{P}^{*} \mathscr{T}^{j} i_{P}\right)(u), v-u\right\rangle+J(v)-J(u) \geq 0,
\end{aligned}
$$

$\forall v \in X$

Step 2. We will prove that $i_{P}^{*} \widetilde{f}_{0} i_{P}$ is a pseudomonotone and bounded mapping from $X$ to $\mathscr{K}\left(X^{*}\right)$.

(i) We prove that $\left(i_{P}^{*} \tilde{f}_{0} i_{P}\right)(u)$ is a nonempty, bounded, closed, and convex subset of $X^{*}$ for all $u \in X$. Moreover, $i_{P}^{*} \widetilde{f}_{0} i_{P}$ is a bounded mapping from $X$ to $\mathscr{K}\left(X^{*}\right)$.

Clearly, for any $u \in L_{P}(\Omega), \widetilde{f}_{0}(u)$ is a nonempty, bounded, closed, and convex subset of $L_{\bar{P}}(\Omega)$; in particular, $\tilde{f}_{0}(u) \in$ $\mathscr{K}\left(L_{\bar{P}}(\Omega)\right)$. Moreover, $\widetilde{f}_{0}$ is a bounded operator from $L_{P}(\Omega)$ to $\mathscr{K}\left(L_{\bar{P}}(\Omega)\right)$.

For any $u \in X$, from the boundedness of $i_{P}^{*}$ and the above arguments, we get that $\left(i_{P}^{*} \tilde{f}_{0} i_{P}\right)(u)=i_{P}^{*} \tilde{f}_{0}(u)$ is a nonempty, bounded, closed, and convex subset of $X^{*}$. Moreover, since $\left\|i_{P}^{*} \eta\right\|_{X^{*}} \leq C\|\eta\|_{L_{\bar{p}}(\Omega)}, \forall \eta \in L_{\bar{P}}(\Omega)$ for some constant $C>$ 0 , it follows from the boundedness of $\tilde{f}_{0}$ that $i_{P}^{*} \widetilde{f}_{0} i_{P}$ is also a bounded mapping. Next, we prove that $\left(i_{P}^{*} \tilde{f}_{0} i_{P}\right)(u)$ is closed in $X^{*}$. In fact, assume $\left\{\eta_{n}\right\} \subset\left(i_{P}^{*} \widetilde{f}_{0} i_{P}\right)(u), \eta_{n}=i_{P}^{*} \tilde{\eta}_{n}$ with $\tilde{\eta}_{n} \in$ $\widetilde{f}_{0}\left(i_{P}(u)\right)=\widetilde{f}_{0}(u), \forall n \in \mathbb{N}$, and

$$
\eta_{n} \longrightarrow \eta \quad \text { in } X^{*}
$$

Because $\left\{\widetilde{\eta}_{n}: n \in \mathbb{N}\right\} \subset \tilde{f}_{0}(u),\left\{\widetilde{\eta}_{n}\right\}$ is a bounded sequence in $L_{\bar{P}}(\Omega)$. By passing to a subsequence if necessary, we can assume without loss of generality that

$$
\tilde{\eta}_{n} \rightarrow \tilde{\eta}_{0} \quad \text { weakly- } * \text { in } L_{\bar{P}}(\Omega) \text { for } \sigma\left(L_{\bar{P}}(\Omega), L_{P}(\Omega)\right) \text {. }
$$

Since $\tilde{f}_{0}(u)$ is weakly convex and closed in $L_{\bar{P}}(\Omega)$ and $L_{P}(\Omega)$ is reflexive, $\tilde{\eta}_{0} \in \tilde{f}_{0}(u)$, and thus $i_{P}^{*} \widetilde{\eta}_{0} \in i_{P}^{*} \widetilde{f}_{0}(u)=\left(i_{P}^{*} \widetilde{f}_{0} i_{P}\right)(u)$. On the other hand, since $i_{P}^{*}$ is continuous from $L_{\bar{p}}(\Omega)$ to $X^{*}$ with both weak topologies, we have from (40) that
$\eta_{n}=i_{P}^{*} \tilde{\eta}_{n} \rightarrow i_{P}^{*} \tilde{\eta}_{0}$ in $X^{*}$ which combined with (39) yields $\eta=i_{P}^{*} \widetilde{\eta}_{0} \in\left(i_{P}^{*} \widetilde{f}_{0} i_{P}\right)(u)$. Hence, $\left(i_{P}^{*} \widetilde{f}_{0} i_{P}\right)(u)$ is closed in $X^{*}$.

(ii) Let $V$ be a finite-dimensional subspace of $X$. We will show that the restriction $\left.\left(i_{P}^{*} \tilde{f}_{0} i_{P}\right)\right|_{V}$ of $i_{P}^{*} \widetilde{f}_{0} i_{P}$ on $V$ is upper semicontinuous from $V$ into $2^{X^{*}}$ with respect to the weak topology on $X^{*}$.

In fact, assume $u_{0} \in V$. To prove the upper semicontinuity of $\left.\left(i_{P}^{*} \widetilde{f}_{0} i_{P}\right)\right|_{V}$ at $u_{0}$, we assume by contradiction that there is a weakly open neighborhood $U$ of $\tilde{f}_{0}\left(u_{0}\right)$ in $X^{*}$ and a sequence $\left\{u_{n}\right\} \subset V$ such that

$$
u_{n} \longrightarrow u_{0} \quad \text { in } V \text {, }
$$

and there exists a sequence $\left\{\eta_{n}\right\} \subset X^{*}$ such that $\eta_{n} \in$ $\left(i_{P}^{*} \tilde{f}_{0} i_{P}\right)\left(u_{n}\right) \backslash U, \forall n \in \mathbb{N}$. We see that $\widetilde{U}=\left(i_{P}^{*}\right)^{-1}(U)$ is a weakly open neighborhood of $\tilde{f}_{0}\left(u_{0}\right)$ in $L_{\bar{P}}(\Omega)$. Moreover, since $\eta_{n} \in\left(i_{P}^{*} \widetilde{f}_{0} i_{P}\right)\left(u_{n}\right)=i_{P}^{*} \tilde{f}_{0}\left(u_{n}\right)$, there exists $\widetilde{\eta}_{n} \in \tilde{f}_{0}\left(u_{n}\right)$ such that

$$
\eta_{n}=i_{P}^{*} \tilde{\eta}_{n}
$$

We have $\tilde{\eta}_{n} \notin \widetilde{U}, \forall n \in \mathbb{N}$. As $\left\{u_{n}\right\}$ is a bounded sequence in $L_{P}(\Omega)$, it follows from (i) that $\left\{\widetilde{\eta}_{n}\right\}$ is a bounded sequence in $L_{\bar{P}}(\Omega)$. Also, as mentioned above, by passing to a subsequence, we can assume that

$$
\widetilde{\eta}_{n} \rightarrow \widetilde{\eta}_{0} \quad \text { weakly in } L_{\bar{P}}(\Omega) \text { for } \sigma\left(L_{\bar{P}}(\Omega), L_{P}(\Omega)\right) \text {, }
$$

for some $\widetilde{\eta}_{0} \in L_{\bar{P}}(\Omega)$. By $(41), u_{n}=i_{P}\left(u_{n}\right) \rightarrow i_{P}\left(u_{0}\right)=u_{0}$ in $L_{P}(\Omega)$. Hence, by passing to a subsequence if necessary, we can assume that

$$
u_{n}(x) \longrightarrow u_{0}(x) \quad \text { for a.e. } x \in \Omega \text {. }
$$

For a.e. $x \in \Omega$ and for any $\varepsilon \in(0,1)$, denote $U(x):=\{\xi \in$ $\left.R: \operatorname{dist}\left(\xi, f_{0}\left(x, u_{0}(x)\right)\right)<\varepsilon\right\}$, where $\operatorname{dist}\left(\xi, f_{0}\left(x, u_{0}(x)\right)\right)=$ $\inf _{z \in f_{0}\left(x, u_{0}(x)\right)}|\xi-z|$. Then, $U(x)$ is open set in $\mathbb{R}$ and $f_{0}\left(x, u_{0}(x)\right) \subset U(x)$. By (F2), there exists $\delta(x)>0$ such that if $\left|v-u_{0}(x)\right|<\delta(x)$ then $f_{0}(x, v) \subset U(x)$. In view of (44), there exists $n_{0}$, such that $\left|u_{n}(x)-u_{0}(x)\right|<\delta(x), \forall n \geq n_{0}$. Hence, $f_{0}\left(x, u_{n}(x)\right) \subset U(x), \forall n \geq n_{0}$. It follows that, for any $z^{\prime} \in f_{0}\left(x, u_{n}(x)\right), \inf _{z \in f_{0}\left(x, u_{0}(x)\right)}\left|z^{\prime}-z\right|<\varepsilon, \forall n \geq n_{0}$. Since $\widetilde{\eta}_{n} \in \tilde{f}_{0}\left(u_{n}\right), \widetilde{\eta}_{n}(x) \in f_{0}\left(x, u_{n}(x)\right)$ for a.e. $x \in \Omega$; thus, there is a sequence $\left\{w_{n}\right\} \subset \widetilde{f}_{0}\left(u_{0}\right)$ such that $\left|\widetilde{\eta}_{n}(x)-w_{n}(x)\right| \rightarrow 0$ as $n \rightarrow \infty$ for a.e. $x \in \Omega$, which implies

$$
\bar{P}\left(\left|\widetilde{\eta}_{n}(x)-w_{n}(x)\right|\right) \longrightarrow 0, \quad \text { for a.e. } x \in \Omega \text {, as } n \longrightarrow \infty \text {. }
$$

On the other hand, since $\bar{P} \in \Delta_{2}$, we have $\bar{P}\left(\left|\widetilde{\eta}_{n}(x)-w_{n}(x)\right|\right) \leq$ $(1 / 2) \bar{P}\left(2\left|\widetilde{\eta}_{n}(x)\right|\right)+(1 / 2) \bar{P}\left(2\left|w_{n}(x)\right|\right) \leq(K / 2) \bar{P}\left(\left|\widetilde{\eta}_{n}(x)\right|\right)+$ $(K / 2) \bar{P}\left(\left|w_{n}(x)\right|\right)$, for some $K>0$. By (32), $\bar{P}\left(\mid \tilde{\eta}_{n}(x)-\right.$ $\left.w_{n}(x) \mid\right) \leq K \bar{P}(h(x))$, for a.e. $x \in \Omega$, all $n \in \mathbb{N}$. Using Lebesgue's dominated convergence theorem, we obtain that

$$
\lim _{n \rightarrow \infty} \int_{\Omega} \bar{P}\left(\left|\widetilde{\eta}_{n}(x)-w_{n}(x)\right|\right) d x=0 .
$$


Since $\bar{P} \in \Delta_{2}$,

$$
\lim _{n \rightarrow \infty}\left\|\widetilde{\eta}_{n}-w_{n}\right\|_{L_{\bar{P}}(\Omega)}=0
$$

In view of (47) and (43), we can deduce that

$$
w_{n} \rightarrow \tilde{\eta}_{0} \quad \text { weakly in } L_{\bar{P}}(\Omega) \text { for } \sigma\left(L_{\bar{P}}(\Omega), L_{P}(\Omega)\right) \text {, }
$$

as $n \rightarrow \infty$. Therefore, $\tilde{\eta}_{0} \in \tilde{f}_{0}\left(u_{0}\right)$ by the convexity and closeness of $\tilde{f}_{0}\left(u_{0}\right)$ and the reflexivity of $L_{P}(\Omega)$. Consequently, $\widetilde{\eta}_{0} \in \widetilde{U}$. By (43) and the reflexivity of $L_{P}(\Omega)$, there exists $N_{0} \in \mathbb{N}$, such that $\tilde{\eta}_{n} \in \widetilde{U}$, for any $n \geq N_{0}$. From (42), $\eta_{n} \in U$; this is a contradiction.

(iii) Referring to [19, Proposition 2.2] we can get that if $\left\{u_{n}\right\} \subset X$ with $u_{n} \rightarrow u_{0}$ and if $\eta_{n} \in\left(i_{P}^{*} \tilde{f}_{0} i_{P}\right)\left(u_{n}\right)$ such that $\lim \sup _{n \rightarrow \infty}\left\langle\eta_{n}, u_{n}-u_{0}\right\rangle \leq 0$, then to each element $v \in X$, $\eta(v) \in\left(i_{P}^{*} \widetilde{f}_{0} i_{P}\right)\left(u_{0}\right)$ exists with

$$
\liminf _{n \rightarrow \infty}\left\langle\eta_{n}, u_{n}-v\right\rangle \geq\left\langle\eta(v), u_{0}-v\right\rangle .
$$

By (i)-(iii) and using Definition 3, we get that $i_{P}^{*} \widetilde{f}_{0} i_{P}$ is a pseudomonotone and bounded mapping from $X$ to $\mathscr{K}\left(X^{*}\right)$.

Step 3. By Step 1 and Step $2, \mathscr{A}+i_{P}^{*} \tilde{f}_{0} i_{P}+i_{M}^{*} \mathscr{B} i_{M}-\sum_{i=1}^{k} i_{P}^{*} \mathscr{T}_{i} i_{P}+$ $\sum_{j=1}^{m} i_{P}^{*} \mathscr{T}^{j} i_{P}$ is a multivalued bounded pseudomonotone mapping on $X$ with domain $D\left(\mathscr{A}+i_{P}^{*} \tilde{f}_{0} i_{P}+i_{M}^{*} \mathscr{B} i_{M}-\right.$ $\left.\sum_{i=1}^{k} i_{P}^{*} \mathscr{T}_{i} i_{P}+\sum_{j=1}^{m} i_{P}^{*} \mathscr{T}^{j} i_{P}\right)=X$.

From the definition of $\partial J(38)$ is equivalent to $0 \in(\mathscr{A}+$ $\left.i_{P}^{*} \tilde{f}_{0} i_{P}+i_{M}^{*} \mathscr{B} i_{M}-\sum_{i=1}^{k} i_{P}^{*} \mathscr{T}_{i} i_{P}+\sum_{j=1}^{m} i_{P}^{*} \mathscr{T}^{j} i_{P}\right)(u)+\partial J(u)$. By [17, Proposition 32.17] or [27, Theorem 4], $\partial J$ is maximal monotone.

Step 4. For $u_{0} \in D(J)$, we check that $\mathscr{A}+i_{P}^{*} \widetilde{f}_{0} i_{P}+i_{M}^{*} \mathscr{B} i_{M}-$ $\sum_{i=1}^{k} i_{P}^{*} \mathscr{T}_{i} i_{P}+\sum_{j=1}^{m} i_{P}^{*} \mathscr{T}^{j} i_{P}$ is $u_{0}$-coercive.

In fact, for any $u \in X$, by (A1), Young inequality, and the convexity of $\bar{M}$, we have

$$
\begin{aligned}
\left\langle\mathscr{A}(u), u-u_{0}\right\rangle \geq & \int_{\Omega} b_{2} M(|D u(x)|) d x \\
& -\int_{\Omega} a_{2}(x) d x-\int_{\Omega} \bar{M}\left(a_{1}(x)\right) d x \\
& -\int_{\Omega} M\left(\left|D u_{0}(x)\right|\right) d x \\
& -b_{1} \int_{\Omega} \bar{M}\left(\varepsilon \bar{M}^{-1}(M(|D u(x)|))\right) d x \\
& -\int_{\Omega} M\left(\frac{1}{\varepsilon}\left|D u_{0}(x)\right|\right) d x \\
\geq & \left(b_{2}-\varepsilon b_{1}\right) \int_{\Omega} M(|D u(x)|) d x-C_{1},
\end{aligned}
$$

where $\varepsilon=\min \left\{b_{2} / 2 b_{1}, 1\right\}$ and $C_{1}$ is a positive constant independent of $u$.
From (32), for any $\tilde{\eta} \in\left(i_{P}^{*} \widetilde{f}_{0} i_{P}\right)(u)$,

$$
\begin{aligned}
\left|\left\langle\widetilde{\eta}, u-u_{0}\right\rangle\right| & \leq\|\widetilde{\eta}\|_{X^{*}} \cdot\left\|u-u_{0}\right\|_{X} \\
& \leq\|\widetilde{\eta}\|_{X^{*}}\left(\|u\|_{X}+\left\|u_{0}\right\|_{X}\right) \\
& \leq C_{2}\left(\|u\|_{X}+1\right),
\end{aligned}
$$

for some constant $C_{2}>0$.

Since $M$ is convex and $M \in \Delta_{2}$, there exists $K>1$ such that $M(|u|) \leq(K / 2)[M(u-\bar{u}(x))+M(|\bar{u}(x)|)]$ whenever $u>\bar{u}(x)$, and $M(|u|) \leq(K / 2)[M(\underline{u}(x)-u)+M(|\underline{u}(x)|)]$ whenever $u<\underline{u}(x)$ for $x \in \Omega, u \in \mathbb{R}$. Note that $\bar{M}(t) \leq$ $|t| \bar{M}^{-1} M(|t|), \forall t \in \mathbb{R}$. In the sequel, we use the set notation $\{u>\bar{u}\}=\{x \in \Omega: u(x)>\bar{u}(x)\},\{\underline{u} \leq u \leq \bar{u}\}=\{x \in \Omega:$ $\underline{u}(x) \leq u(x) \leq \bar{u}(x)\}$, and $\{u<\underline{u}\}=\{x \in \Omega: u(x)<\underline{u}(x)\}$. Then we have

$$
\begin{aligned}
& \left\langle\left(i_{M}^{*} \mathscr{B} i_{M}\right)(u), u\right\rangle \\
& =\langle\mathscr{B}(u), u\rangle_{L_{\bar{P}}(\Omega), L_{P}(\Omega)} \\
& =\int_{\Omega} b(x, u(x)) u(x) d x \\
& =\int_{\{u>\bar{u}\}} \bar{M}^{-1} M(u(x)-\bar{u}(x))(u(x)-\bar{u}(x)) d x \\
& +\int_{\{u>\bar{u}\}} \bar{M}^{-1} M(u(x)-\bar{u}(x)) \bar{u}(x) d x \\
& +\int_{\{u<\underline{u}\}} \bar{M}^{-1} M(\underline{u}(x)-u(x))(\underline{u}(x)-u(x)) d x \\
& -\int_{\{u<\underline{u}\}} \bar{M}^{-1} M(\underline{u}(x)-u(x)) \underline{u}(x) d x \\
& \geq \int_{\{u>\bar{u}\}} M(u(x)-\bar{u}(x)) d x \\
& -\int_{\{u>\bar{u}\}} \bar{M}\left(\varepsilon_{1} \bar{M}^{-1} M(u(x)-\bar{u}(x))\right) d x \\
& -\int_{\{u>\bar{u}\}} M\left(\frac{1}{\varepsilon_{1}} \bar{u}(x)\right) d x+\int_{\{u<\underline{u}\}} M(\underline{u}(x)-u(x)) d x \\
& -\int_{\{u<\underline{u}\}} \bar{M}\left(\varepsilon_{1} \bar{M}^{-1} M(\underline{u}(x)-u(x))\right) d x \\
& -\int_{\{u<\underline{u}\}} M\left(\frac{1}{\varepsilon_{1}} \underline{u}(x)\right) d x \\
& \geq\left(1-\varepsilon_{1}\right) \int_{\{u>\bar{u}\}} M(u(x)-\bar{u}(x)) d x \\
& +\left(1-\varepsilon_{1}\right) \int_{\{u<\underline{u}\}} M(\underline{u}(x)-u(x)) d x-C_{3} \\
& \geq\left(1-\varepsilon_{1}\right)\left\{\int_{\{u>\bar{u}\}}\left[\frac{2}{K} M(u(x))-M(\bar{u}(x))\right] d x\right.
\end{aligned}
$$




$$
\begin{aligned}
& \left.+\int_{\{u<\underline{u}\}}\left[\frac{2}{K} M(u(x))-M(\underline{u}(x))\right] d x\right\}-C_{3} \\
& \geq\left(1-\varepsilon_{1}\right) \frac{2}{K}\left\{\int_{\{u>\bar{u}\}} M(u(x)) d x+\int_{\{u<\underline{u}\}} M(u(x)) d x\right\} \\
& +\left(1-\varepsilon_{1}\right) \frac{2}{K} \\
& \cdot\left\{\int_{\{\underline{u} \leq u \leq \bar{u}\}}[M(u(x))-M(|\underline{u}| \vee|\bar{u}|(x))] d x\right\}-C_{4} \\
& \geq\left(1-\varepsilon_{1}\right) \frac{2}{K} \int_{\Omega} M(u(x)) d x-C_{5}, \\
& \left|\left\langle\left(i_{M}^{*} \mathscr{B} i_{M}\right)(u), u_{0}\right\rangle\right| \\
& \leq \int_{\Omega}|b(x, u(x))| \cdot\left|u_{0}(x)\right| d x \\
& =\int_{\{u>\bar{u}\}} \bar{M}^{-1} M(u(x)-\bar{u}(x)) \cdot\left|u_{0}(x)\right| d x \\
& +\int_{\{u<u\}} \bar{M}^{-1} M(\underline{u}(x)-u(x)) \cdot\left|u_{0}(x)\right| d x \\
& \leq \int_{\{u>\bar{u}\}} \varepsilon_{3} M((u(x)-\bar{u}(x))) d x \\
& +\int_{\{u>\bar{u}\}} M\left(\frac{1}{\varepsilon_{3}}\left|u_{0}(x)\right|\right) d x \\
& +\int_{\{u<u\}} \varepsilon_{3} M((\underline{u}(x)-u(x))) d x \\
& +\int_{\{u<\underline{u}\}} M\left(\frac{1}{\varepsilon_{3}}\left|u_{0}(x)\right|\right) d x \\
& \leq \frac{K \varepsilon_{3}}{2} \int_{\Omega} M(u(x)) d x+\frac{K \varepsilon_{3}}{2} \int_{\Omega} M(\bar{u}(x)) d x \\
& +\frac{K \varepsilon_{3}}{2} \int_{\Omega} M(\underline{u}(x)) d x+\int_{\Omega} M\left(\frac{1}{\varepsilon_{3}}\left|u_{0}(x)\right|\right) d x \\
& \leq \frac{K \varepsilon_{3}}{2} \int_{\Omega} M(u(x)) d x+C_{6},
\end{aligned}
$$

where $\varepsilon_{1}, \varepsilon_{2} \in(0,1), C_{3}, C_{4}, C_{5}, C_{6}>0$. Taking $\varepsilon_{1}=1 / 2$ and $\varepsilon_{2}=1 / K^{2}$, we can deduce that

$$
\begin{aligned}
& \left\langle\left(i_{M}^{*} \mathscr{B} i_{M}\right)(u), u-u_{0}\right\rangle \\
& \quad \geq\left\langle\left(i_{M}^{*} \mathscr{B} i_{M}\right)(u), u\right\rangle-\left|\left\langle\left(i_{M}^{*} \mathscr{B} i_{M}\right)(u), u_{0}\right\rangle\right| \\
& \geq\left[\left(1-\varepsilon_{1}\right) \frac{2}{K}-\frac{K \varepsilon_{3}}{2}\right] \int_{\Omega} M(u(x)) d x-C_{7} \\
& \quad=\frac{1}{2 K} \int_{\Omega} M(u(x)) d x-C_{7} .
\end{aligned}
$$

For $i \in\{1, \ldots, k\}$, we have

$$
\begin{aligned}
& \left|\left\langle\left(i_{P}^{*} \mathscr{T}_{i} i_{P}\right)(u), u-u_{0}\right\rangle\right| \\
& \quad=\left|\int_{\Omega} T_{i}(x, u(x))\left(u(x)-u_{0}(x)\right) d x\right| \\
& \quad \leq\left\|\underline{\eta}_{i}-\underline{\eta}\right\|_{L_{\bar{P}(\Omega)}}\|u\|_{L_{P(\Omega)}}+\left\|\underline{\eta}_{i}-\underline{\eta}\right\|_{L_{\bar{P}(\Omega)}}\left\|u_{0}\right\|_{L_{P(\Omega)}},
\end{aligned}
$$

and thus

$$
\sum_{i=1}^{k}\left|\left\langle\left(i_{P}^{*} \mathscr{T}_{i} i_{P}\right)(u), u-u_{0}\right\rangle\right| \leq C_{8}\left(\|u\|_{X}+1\right),
$$

for some $C_{8}>0$. Similarly,

$$
\sum_{j=1}^{m}\left|\left\langle\left(i_{P}^{*} \mathscr{T}^{j} i_{P}\right)(u), u-u_{0}\right\rangle\right| \leq C_{9}\left(\|u\|_{X}+1\right),
$$

for some $C_{9}>0$.

Define, for $u \in X, \rho(u)=\int_{\Omega}[M(|D u(x)|)+M(|u(x)|)] d x$ and $\|u\|_{\rho}=\inf \{\lambda>0: \rho(u / \lambda) \leq 1\}$. Then, it is easy to see that $(1 / 2)\|u\|_{\rho} \leq\|u\|_{X} \leq 2\|u\|_{\rho}$.

Let $\|u\|_{X} \rightarrow+\infty$. Then $\|u\|_{\rho} \rightarrow+\infty$. Combining the estimates from (50)-(56) and using (8), we obtain that

$$
\begin{aligned}
& \left\langle\mathscr{A}(u)+\widetilde{\eta}+\left(i_{M}^{*} \mathscr{B} i_{M}\right)(u)-\sum_{i=1}^{k}\left(i_{P}^{*} \mathscr{T}_{i} i_{P}\right)(u)\right. \\
& \left.+\sum_{j=1}^{m}\left(i_{P}^{*} \mathscr{T}^{j} i_{P}\right)(u), u-u_{0}\right\rangle \\
& \cdot\left(\|u\|_{X}\right)^{-1} \\
& \geq\left\langle\mathscr{A}(u)+\widetilde{\eta}+\left(i_{M}^{*} \mathscr{B} i_{M}\right)(u)-\sum_{i=1}^{k}\left(i_{P}^{*} \mathscr{T}_{i} i_{P}\right)(u)\right. \\
& \left.+\sum_{j=1}^{m}\left(i_{P}^{*} \mathscr{T}^{j} i_{P}\right)(u), u-u_{0}\right\rangle \\
& \cdot\left(2\|u\|_{\rho}\right)^{-1} \\
& \geq \frac{1}{2\|u\|_{\rho}}\left[\left(b_{2}-\varepsilon b_{1}\right) \int_{\Omega} M(|D u(x)|) d x\right. \\
& \left.\quad+\frac{1}{2 K} \int_{\Omega} M(u(x)) d x\right]-C_{10}-\frac{C_{11}}{\|u\|_{\rho}} \\
& \quad+\frac{C_{12}}{\|u\|_{\rho}}\left[\int_{\Omega} M\left(\left(\|u\|_{\rho}-\varepsilon\right) \frac{|\nabla u(x)|}{\|u\|_{\rho}-\varepsilon}\right) d x\right. \\
& \left.-C_{10}-\frac{C_{11}}{\|u\|_{\rho}}\left(\left(\|u\|_{\rho}-\varepsilon\right) \frac{|u(x)|}{\|u\|_{\rho}-\varepsilon}\right) d x\right]
\end{aligned}
$$




$$
\begin{aligned}
\geq & \frac{C_{12}}{\|u\|_{\rho}}\left(\|u\|_{\rho}-\varepsilon\right) g\left(\|u\|_{\rho}-\varepsilon\right) \\
& \cdot\left[\int_{\Omega} M\left(\frac{|D u(x)|}{\|u\|_{\rho}-\varepsilon}\right) d x+\int_{\Omega} M\left(\frac{|u(x)|}{\|u\|_{\rho}-\varepsilon}\right) d x\right] \\
& -C_{10}-\frac{C_{11}}{\|u\|_{\rho}} \\
\geq & \frac{C_{12}}{\|u\|_{\rho}}\left(\|u\|_{\rho}-\varepsilon\right) g\left(\|u\|_{\rho}-\varepsilon\right)-C_{10}-\frac{C_{11}}{\|u\|_{\rho}} .
\end{aligned}
$$

By the arbitrary of $\varepsilon$, we have

$$
\begin{aligned}
& \left\langle\mathscr{A}(u)+\tilde{\eta}+\left(i_{M}^{*} \mathscr{B} i_{M}\right)(u)-\sum_{i=1}^{k}\left(i_{P}^{*} \mathscr{T}_{i} i_{P}\right)(u)\right. \\
& \left.+\sum_{j=1}^{m}\left(i_{P}^{*} \mathscr{T}^{j} i_{P}\right)(u), u-u_{0}\right\rangle \\
& \cdot\left(\|u\|_{X}\right)^{-1} \\
& \geq \frac{C_{12}}{\|u\|_{\rho}}\|u\|_{\rho} g\left(\|u\|_{\rho}\right)-C_{10}-\frac{C_{11}}{\|u\|_{\rho}} \\
& =C_{12} g\left(\|u\|_{\rho}\right)-C_{10}-\frac{C_{11}}{\|u\|_{\rho}} .
\end{aligned}
$$

Hence,

$$
\begin{aligned}
& \lim _{\|u\|_{X} \rightarrow+\infty}\left[\inf _{\tilde{\eta} \in F_{0}(u)}(\langle\mathscr{A}(u)+\widetilde{\eta}\right. \\
&+\left(i_{M}^{*} \mathscr{B} i_{M}\right)(u) \\
&-\sum_{i=1}^{k}\left(i_{P}^{*} \mathscr{T}_{i} i_{P}\right)(u) \\
&\left.+\sum_{j=1}^{m}\left(i_{P}^{*} \mathscr{T}^{j} i_{P}\right)(u), u-u_{0}\right\rangle \\
&\left.\left.\cdot\left(\|u\|_{X}\right)^{-1}\right)\right] \\
&=+\infty,
\end{aligned}
$$

where $F_{0}(u):=\left(i_{P}^{*} \tilde{f}_{0} i_{P}\right)(u)$. Therefore, $\mathscr{A}+i_{P}^{*} \widetilde{f}_{0} i_{P}+i_{M}^{*} \mathscr{B} i_{M}-$ $\sum_{i=1}^{k} i_{P}^{*} \mathscr{T}_{i} i_{P}+\sum_{j=1}^{m} i_{P}^{*} \mathscr{T}^{j} i_{P}$ is $u_{0}$-coercive.

We have verified that $\mathscr{A}+i_{P}^{*} \widetilde{f}_{0} i_{P}+i_{M}^{*} \mathscr{B} i_{M}-\sum_{i=1}^{k} i_{P}^{*} \mathscr{T}_{i} i_{P}+$ $\sum_{j=1}^{m} i_{P}^{*} \mathscr{T}^{j} i_{P}+\partial J$ satisfy the conditions of Lemma 5 . By Lemma $5, \mathscr{A}+i_{P}^{*} \tilde{f}_{0} i_{P}+i_{M}^{*} \mathscr{B} i_{M}-\sum_{i=1}^{k} i_{P}^{*} \mathscr{T}_{i} i_{P}+\sum_{j=1}^{m} i_{P}^{*} \mathscr{T}^{j} i_{P}+\partial J$ is surjective, that is, $R\left(\mathscr{A}+i_{P}^{*} \tilde{f}_{0} i_{P}+i_{M}^{*} \mathscr{B} i_{M}-\sum_{i=1}^{k} i_{P}^{*} \mathscr{T}_{i} i_{P}+\right.$ $\left.\sum_{j=1}^{m} i_{P}^{*} \mathscr{T}^{j} i_{P}+\partial J\right)=X^{*}$. Hence, (38) has a solution $u$.

Step 5. Let $u$ be any solution of (38). Following the lines in [1], we can deduce (28).
From (28) and the definitions of $b, T_{i}$ and $T^{j}$, we have $b(\cdot, u)=T_{i}(\cdot, u)=T^{j}(\cdot, u)$ a.e in $\Omega$, for all $i \in\{1, \ldots, k\}, j \in$ $\{1, \ldots, m\}$. Also, we get that $\eta(x) \in f_{0}(x, u(x))=f(x, u(x))$ for a.e. $x \in \Omega$. In view of these observations, (38) reduces to (25). Our proof of Theorem 11 is complete.

The choice $M(u)=|u|^{p}, p>1$ in Theorem 11 leads to Theorem 2.11 in [1].

Under the assumptions of Theorem 11, we define

$\mathcal{S}=\{u \in X: u$ is a solution of (9), $\underline{u} \leq u \leq \bar{u}$ a.e. in $\Omega\}$,

and then the set $\mathcal{S}$ is nonempty. As consequences of Theorem 11, some further properties of $\delta$ are given in the following corollary. The proofs of these properties are omitted, since they do not require substantial modifications as in Theorem 11.

Corollary 12. Under the assumptions of Theorem 11, the following assertions are true.

(i) Any $u \in \mathcal{S}$ is both a subsolution and a supersolution of (9).

(ii) $\mathcal{S}$ is directed downward and upward; that is, for all $u_{1}, u_{2} \in \mathcal{S}$, there exist $u_{3}, u_{4} \in \mathcal{S}$ such that $u_{3} \leq u_{1} \wedge u_{2}$ and $u_{4} \geq u_{1} \vee u_{2}$.

Remark 13. If $v \in W_{0}^{1} L_{M}(\Omega)$ in problem (P), then the condition (8) can be omitted. In fact, by Lemma 3.14 and Lemma 5.7 in [15] the coerciveness holds in Step 4 of the proof of Theorem 11.

Moreover, if $J$ is given by an integral functional such as $J(u)=\int_{\Omega} M(|D u|) d x, F(u)$ is a single-valued term, then problem (P) implies problem (1.1) in [28].

\section{Conflict of Interests}

The authors declare that there is no conflict of interests regarding the publication of this paper.

\section{Acknowledgments}

The authors are highly grateful for the referee's careful reading and comments on this paper. The first author was supported by "Chen Guang" Project (supported by Shanghai Municipal Education Commission and Shanghai Education Development Foundation) (10CGB25) and Shanghai Colleges for Outstanding Young Teachers' Scientific Research Selection and Training Special Fund (sjq08011). The second author was supported by the National Natural Science Foundation of China (11371279).

\section{References}

[1] V. K. Le, "On variational and quasi-variational inequalities with multivalued lower order terms and convex functionals," Nonlinear Analysis: Theory, Methods \& Applications, vol. 94, pp. 12-31, 2014. 
[2] V. K. Le, "On variational inequalities with maximal monotone operators and multivalued perturbing terms in Sobolev spaces with variable exponents," Journal of Mathematical Analysis and Applications, vol. 388, no. 2, pp. 695-715, 2012.

[3] V. K. Le, "A range and existence theorem for pseudomonotone perturbations of maximal monotone operators," Proceedings of the American Mathematical Society, vol. 139, no. 5, pp. 16451658, 2011.

[4] S. Carl and V. K. Le, "Quasilinear parabolic variational inequalities with multi-valued lower-order terms," Zeitschrift für angewandte Mathematik und Physik, vol. 65, no. 5, pp. 845-864, 2014.

[5] X. L. Fan, "Differential equations of divergence form in Musielak-Sobolev spaces and a sub-supersolution method," Journal of Mathematical Analysis and Applications, vol. 386, no. 2, pp. 593-604, 2012.

[6] L. Aharouch and J. Bennouna, "Existence and uniqueness of solutions of unilateral problems in Orlicz spaces," Nonlinear Analysis: Theory, Methods \& Applications, vol. 72, no. 9-10, pp. 3553-3565, 2010.

[7] G. Dong and Z. Shi, "An existence theorem for weak solutions for a class of elliptic partial differential systems in Orlicz spaces," Nonlinear Analysis: Theory, Methods \& Applications, vol. 68, no. 4, pp. 1037-1042, 2008.

[8] G. Dong, "An existence theorem for weak solutions for a class of elliptic partial differential systems in general Orlicz-Sobolev spaces," Nonlinear Analysis: Theory, Methods \& Applications, vol. 69, no. 7, pp. 2049-2057, 2008.

[9] G. Dong, "Elliptic equations with measure data in Orlicz spaces," Electronic Journal of Differential Equations, vol. 76, pp. 1-10, 2008.

[10] X. C. Fang, E. R. Hou, and G. Dong, "Solutions to the system of operator equations $A_{1} X=C_{1}, X B_{2}=C_{2}$, and $A_{3} X B_{3}=C_{3}$ on Hilbert $C^{*}$-modules," Abstract and Applied Analysis, vol. 2013, Article ID 826564, 8 pages, 2013.

[11] X. Fang and J. Yu, "Solutions to operator equations on Hilbert $C^{*}$-modules II," Integral Equations and Operator Theory, vol. 68, no. 1, pp. 23-60, 2010.

[12] X. C. Fang, J. Yu, and H. Yao, "Solutions to operator equations on Hilbert $C^{*}$-modules," Linear Algebra and its Applications, vol. 431, no. 11, pp. 2142-2153, 2009.

[13] M. Krasnoselski and Y. Rutickii, Convex Functions and Orlicz Spaces, Noordhoff, Groningen, The Netherlands, 1961.

[14] R. A. Adams and J. J. F. Fournier, Sobolev Spaces, vol. 140, Academic Press, New York, NY, USA, 2nd edition, 2003.

[15] J.-P. Gossez, "Nonlinear elliptic boundary value problems for equations with rapidly (or slowly) increasing coefficients," Transactions of the American Mathematical Society, vol. 190, pp. 163-205, 1974.

[16] S. T. Chen, "Geometry of orlicz spaces," Dissertationes Mathematicae, vol. 356, 1996.

[17] E. Zeidler, Nonlinear Functional Analysis and Its Applications: II/B: Nonlinear Monotone Operators, Springer, New York, NY, USA, 1990.

[18] S. Carl, V. K. Le, and D. Motreanu, Nonsmooth Variational Problems and Their Inequalities: Comparison Principles and Applications, Springer Monographs in Mathematics, Springer, New York, NY, USA, 2007.

[19] Z. Naniewicz and P. D. Panagiotopoulos, Mathematical Theory of Hemivariational Inequalities and Applications, Marcel Dekker, New York, NY, USA, 1995.
[20] F. E. Browder and P. Hess, "Nonlinear mappings of monotone type in Banach spaces," Journal of Functional Analysis, vol. 11, pp. 251-294, 1972.

[21] J. F. Rodrigues and R. Teymurazyan, "On the two obstacles problem in Orlicz-Sobolev spaces and applications," Complex Variables and Elliptic Equations, vol. 56, no. 7-9, pp. 769-787, 2011.

[22] N. Bourbaki, Integration I, (translated by S. Berberian), Springer, Berlin, Germany, 2004.

[23] S. C. Hu and N. S. Papageorgiou, Handbook of Multivalued Analysis. Vol. I, vol. 419 of Mathematics and Its Applications, Kluwer Academic Publishers, Dodrecht, The Netherlands, 1997.

[24] Y. G. Borisovich, B. D. Gel'man, A. D. Myshkis, and V. V. Obukhovskii, "Multivalued mappings," Journal of Soviet Mathematics, vol. 24, no. 6, pp. 719-791, 1984.

[25] M. García-Huidobro, V. K. Le, R. Manasevich, and K. Schmitt, "On principal eigenvalues for quasilinear elliptic differential operators: an Orlicz-Sobolev space setting," Nonlinear Differential Equations and Applications, vol. 6, no. 2, pp. 207-225, 1999.

[26] V. K. Le, "Variational inequalities with general multivalued lower order terms given by integrals," Advanced Nonlinear Studies, vol. 11, no. 1, pp. 1-24, 2011.

[27] R. T. Rockafellar, "Characterization of the subdifferentials of convex functions," Pacific Journal of Mathematics, vol. 17, pp. 497-510, 1966.

[28] V. K. Le, "Sub-supersolutions in a variational inequality related to a sandpile problem," The ANZIAM Journal, vol. 48, no. 2, pp. 179-197, 2006. 


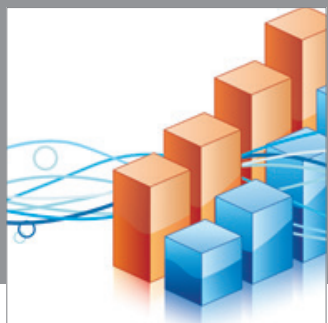

Advances in

Operations Research

mansans

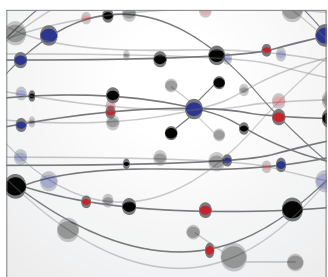

The Scientific World Journal
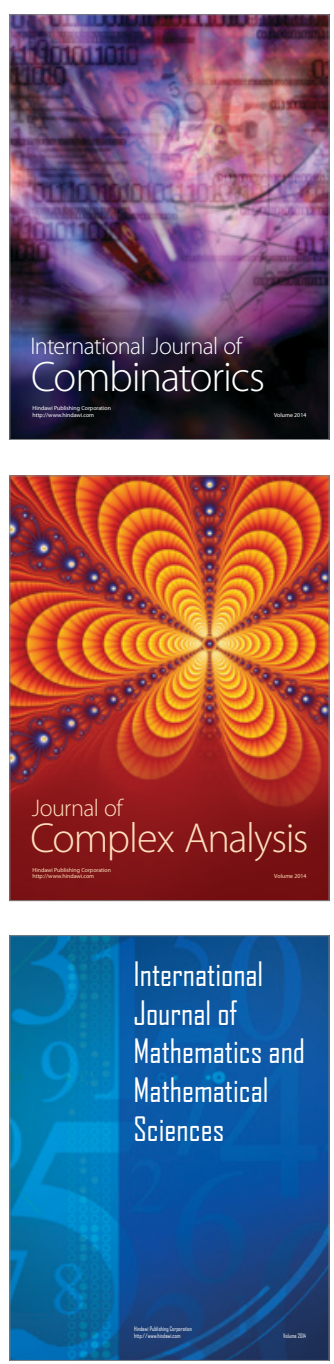
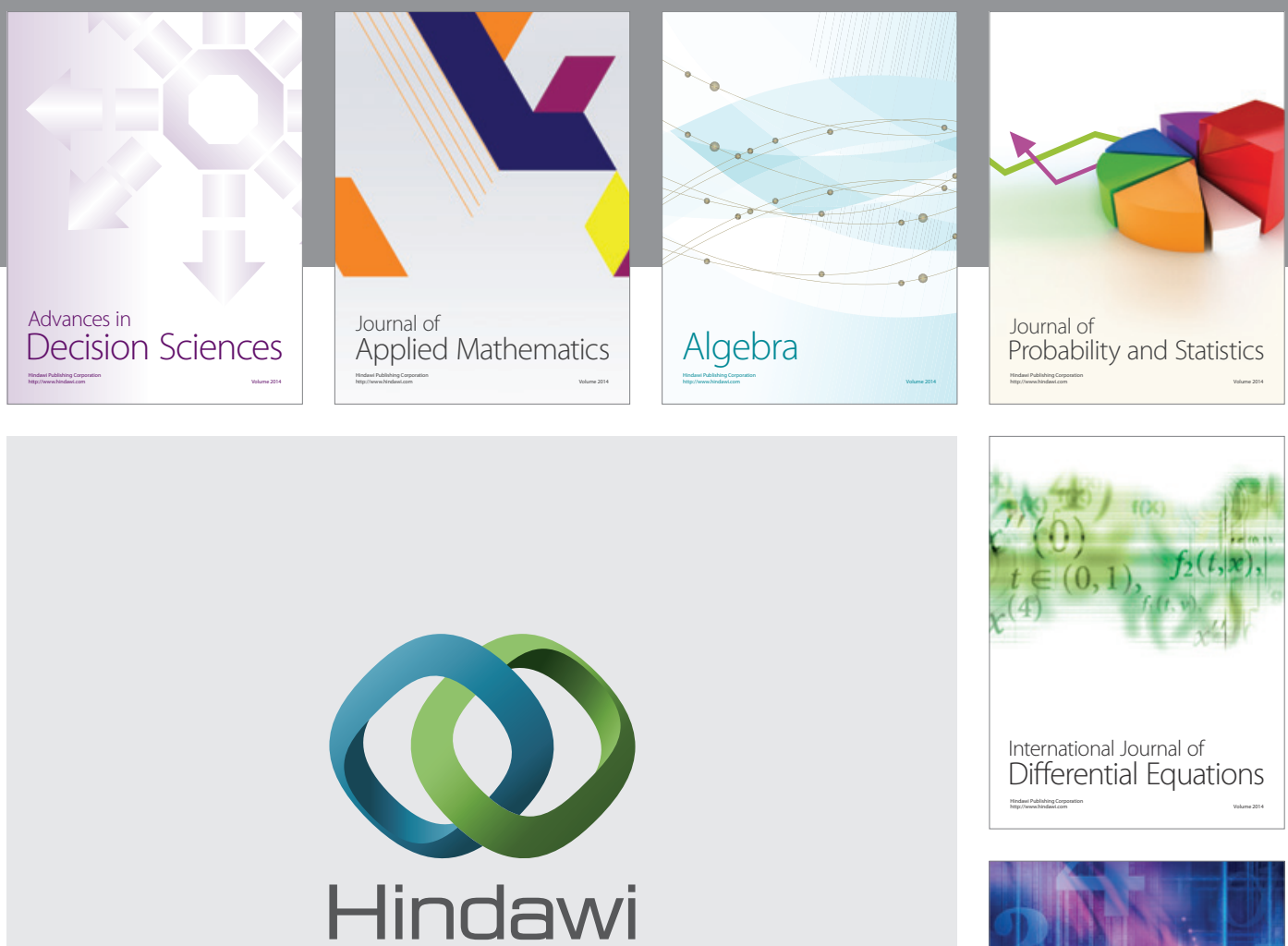

Submit your manuscripts at http://www.hindawi.com
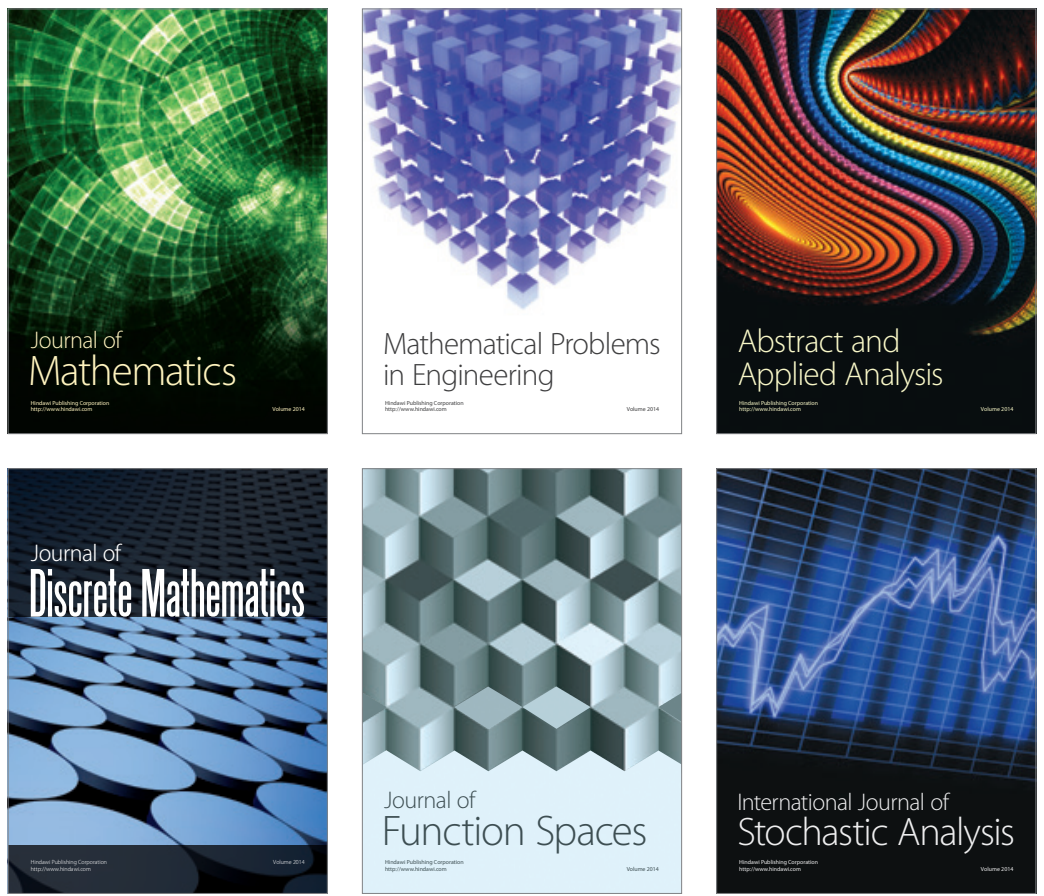

Journal of

Function Spaces

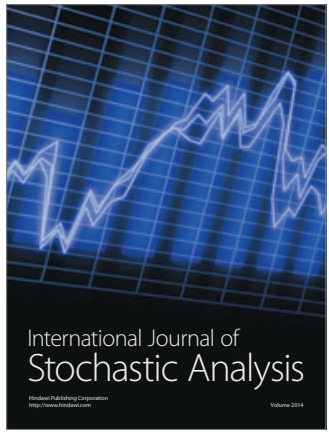

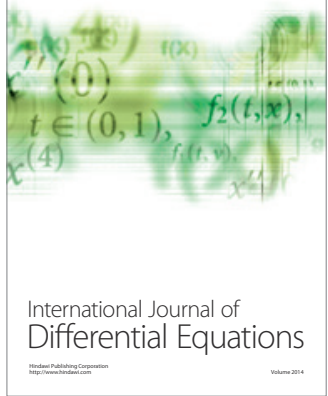
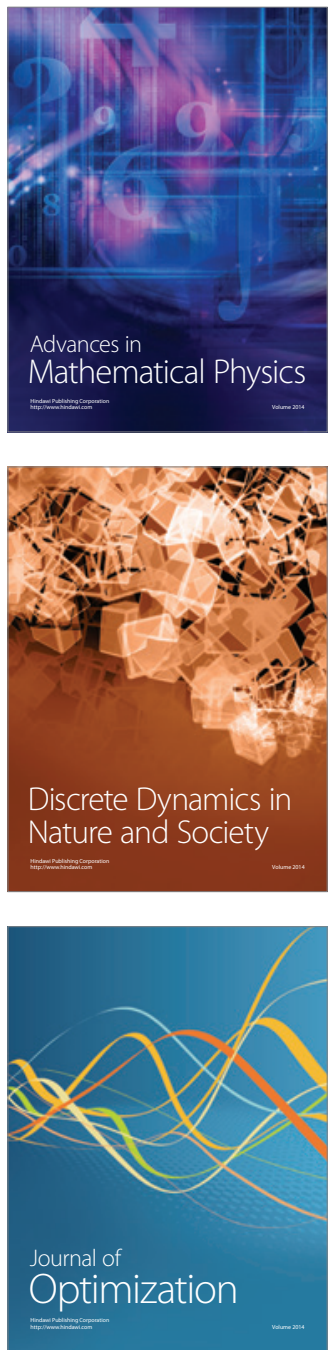